\title{
The ARICH detector at Belle II experiment
}

\author{
Yosuke Yusa*t \\ Niigata University \\ E-mail: yusa@hep.sc.niigata-u.ac.jp
}

We present overview of Aerogel Ring Imaging Cherenkov counter (ARICH) which is developed as a particle identification detector in next generation $B$-factory experiment, the Belle II. The ARICH consists of aerogel radiator and photon sensor. When charged particle goes through the radiator, it emits Cherenkov light to the direction which depends on the particle mass. Using this dependence, we identify the charged particle by reproducing the ring image with high sensitive photon sensor array. We develop new photon sensor named Hybrid Avalanche Photon Detector (HAPD) to collect a small number of Cherenkov photon distributed in large area effectively. We set up small part of ARICH detector and perform the measurement using electron and hadron beam line at KEK, CERN and DESY. From the obtained results, we expect that it is possible to separate $K$ and $\pi$ by more than $4 \sigma$ deviation level with current design of the ARICH. Mass production of HAPD and readout electronics has been started and ARICH will be constructed from this year. Installation to the Belle II detector will be completed in 2015.

The European Physical Society Conference on High Energy Physics -EPS-HEP2013

18-24 July 2013

Stockholm, Sweden

\footnotetext{
${ }^{*}$ Speaker.

${ }^{\dagger}$ on behalf of Belle II collaboration
} 


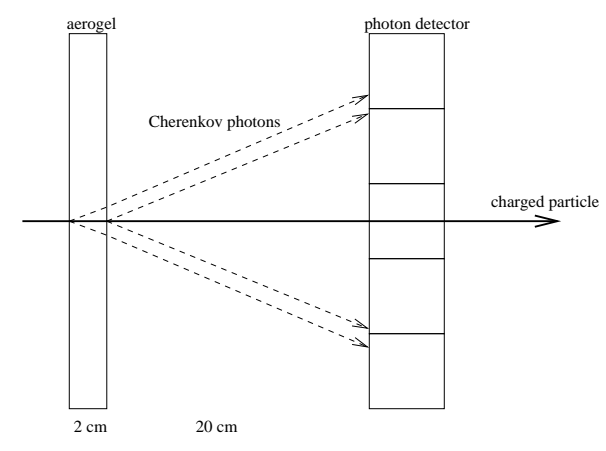

Figure 1: Overview of ARICH detector.

\section{Introduction}

The Belle II experiment is next generation $B$-factory experiment in KEK, Tsukuba Japan [1]. Through asymmetric collision of $e^{+}$and $e^{-}$, a large number of $B$ mesons are produced and many kinds of studies such as precise measurement of Standard Model parameters, new physics search and so on are performed by analyzing various kinds of $B$ meson decays. To reconstruct $B$ meson properly in such studies, it is essential to identify the particles, especially $K$ and $\pi$, that are from $B$ meson decays with high accuracy and efficiency. To achieve that, we plan to install ring shape detector so called "Aerogel Ring Imaging Cherenkov counter" (ARICH) to cover forward region of $e^{+} e^{-}$interaction point. The ARICH consists of array of Cherenkov light radiators, photon sensors and readout electronics. As a charged particle pass through the radiator, it emits Cherenkov light and the photons position information is collected by photon sensor and readout electronics. The angle between Cherenkov photons along to the charged particle path $\theta_{\mathrm{C}}$ (Cherenkov angle) depends on the refraction index of the radiator $n$ and velocity of the charged particle $\beta$ :

$$
\cos \theta_{\mathrm{C}}=\frac{1}{n \beta}
$$

so that we can identify the particle by measuring $\theta_{C}$ from photon hit position information and charged particle direction obtained by drift chamber that is installed in front of the ARICH as shown in Figure 1. Since the difference of Cherenkov angle between $K$ and $\pi$ is expected to be $23 \mathrm{mrad}$ for the momentum of $4 \mathrm{GeV} / \mathrm{c}$, we need to measure photon hit position with high accuracy. Due to the limitation of the space in Belle II detector, we can not install focusing mirror as with Cherenkov ring imaging counters ever used in other experiments. Therefore photon sensor is needed to be high sensitive one to collect Cherenkov photons that are distributed in large area as single photon.

\section{Hybrid Avalanche Photon Detector (HAPD)}

\subsection{Detector overview}

The number of Cherenkov photon emitted from a charged track is expected to be less than 10 . To collect such small number of photon distributed in large area with high position resolution, we 


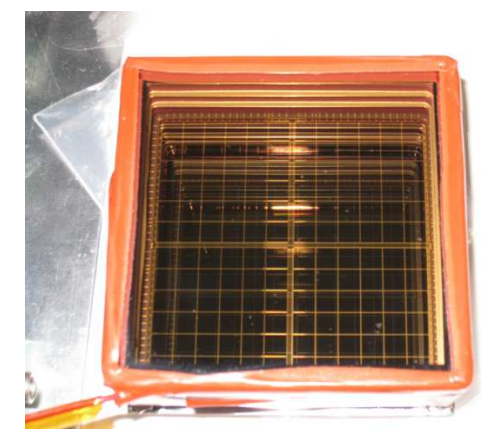

Figure 2: Picture of HAPD.

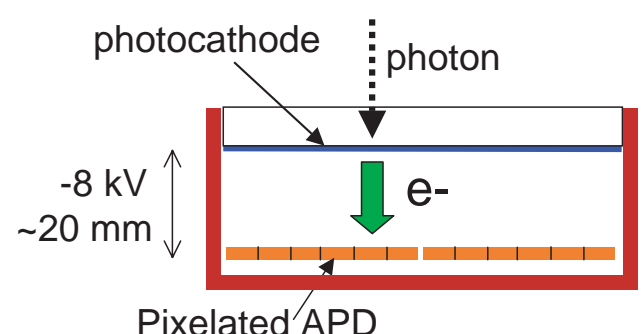

Figure 3: Scheme of photon detection with HAPD.

develop new photon detector called HAPD with Hamamatsu photonics K.K. The HAPD consists of bi-alkali photocathode and $5 \mathrm{~mm}$ pitch pixelated 144 channels avalanche photodiode (APD). Figure 2 and 3 are picture of HAPD and overview of HAPD structure, respectively. A photon is converted to photoelectron at photocathode and it is accelerated by electric field that is induced from high voltage $(-8 \mathrm{kV})$ applied between photocathode and APD. In the APD, the pulse is amplified by electron avalanche. From this hybrid amplification process, we achieve enough gain to observe single photon. Typical gains of bombardment and APD are 1500 and 50, respectively so that total gain reaches about $7.0 \times 10^{4}$.

\subsection{Radiation hardness test}

In operation of the Belle II experiment, we expect high irradiation of background $\gamma$-ray and neutron to the detector. At the region where ARICH is installed (about $1 \mathrm{~m}$ away from $e^{+} e^{-}$ interaction point), it is expected that $1000 \mathrm{~Gy} \gamma$-ray and $1.0 \times 10^{12}$ neutrons $/ \mathrm{cm}^{2}$ will be irradiated in 10 years. The APD is expected to be damaged both from $\gamma$-ray and neutron. When $\gamma$-ray is irradiated, charge-up occurs around the structure on APD surface and breakdown happens finally. Neutron induce lattice defects in silicon bulk and leakage current increases. As a result, signalto-noise ratio becomes worse and photon detection performance degrades. We produce several kind of prototype of APD by changing its material and design (surface structure arrangement, semiconductor layer thickness and so on) and perform radiation hardness test for them using $\gamma$ ray from ${ }^{60} \mathrm{Co}$ radioisotope and neutron from reactor and accelerator. For $\gamma$-ray, we check dark current by applying bias HV to APD during the irradiation and check whether breakdown occurs 


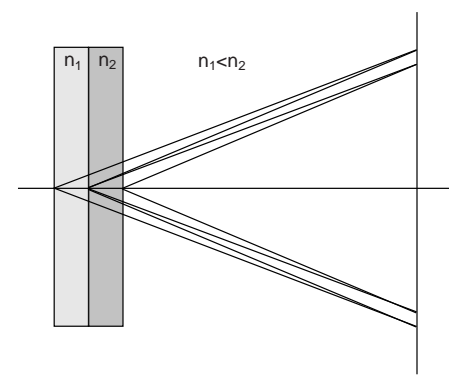

Figure 4: Overview of focusing scheme with 2 aerogel tiles.

or not. For neutron, we measure output pulse for 1 photon electron light source before and after irradiation and check signal-to-noise ratio. After removing alkali-protection layer on APD and changing material of film on active area, breakdown does not occur after irradiating 1000 Gy $\gamma$ ray. When using thin $\mathrm{P}$ and $\mathrm{P}+$ layer for $\mathrm{APD}$, signal-to-noise ratio improves within operable level for particle identification after irradiation more than $10^{12}$ neutrons $/ \mathrm{cm}^{2}$. Furthermore, it is confirmed that the performance is improved by increasing HV between photocathode and APD and optimization of data handling parameters setting in front-end electronics.

\section{Aerogel radiator}

Due to limitation of the space where the ARICH is installed, distance between the Cherenkov light radiator surface of upstream and HAPD photocathode window is set to be $200 \mathrm{~mm}$ and thus refractive index of the radiator should be 1.04-1.06. To cover large area with the materials of such refractive index, we use tiles of silica aerogel. To increase photon yield by focusing without degrading the single photon resolution, we set 2 layers of aerogels with different refractive indices along charged particle incident direction as shown in Figure 4. To suppress photon scattering in the radiator, it is needed to be transparent. Target transmission length is larger than $40 \mathrm{~mm}$ for photons of wavelength equals $400 \mathrm{~nm}$. To produce high transparent and large refractive index aerogel, we study new technique by introducing so called "pin-hole drying" process. A number of samples that satisfies the requirements are obtained by experimental production.

\section{Front-end electronics}

Signal pulse from HAPD is converted to hit information in readout electronics board attached to back side of HAPD. On the board, ASIC that is consist of pre-amplifier, shaper and discriminator is located. There are 36 inputs from HAPD channels in 1 ASIC, thus 4 ASICs are installed on 1 board. Parameters for ASIC operation such as gain, shaping time, offset level and so on are controlled by a FPGA located on the board. Hit information from ASIC is sent to the FPGA and stored in shift register. Stored data is transported to back-end Belle II global DAQ system via optical link in response to the trigger input. The design of front-end electronics is almost finalized and production of the boards that are actually used in the experiment will start soon. 


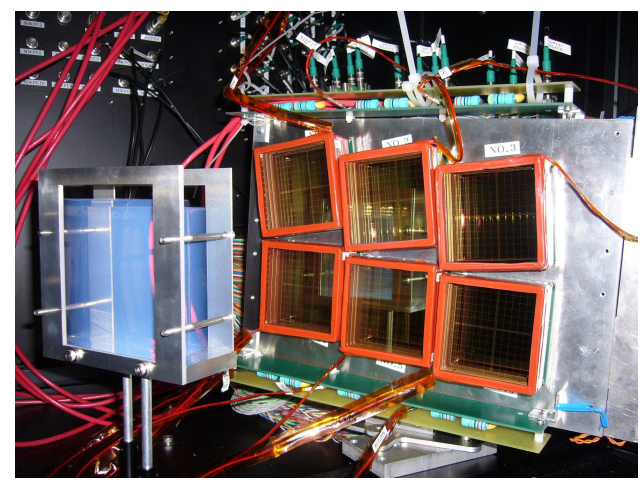

Figure 5: Setup of beam test.

\section{Beam test}

To check performance of the particle identification with actual detector and investigate best solution of detector setup, we construct small part of ARICH using aerogel radiator, 6 HAPDs and readout electronics and perform beam tests, Fuji beam area at KEK in 2009 (3 GeV/c electron beam), SPS H6 beam line at CERN in 2011 (120 GeV/c hadron beam) and T24 beam area at DESY in 2013 (4 GeV/c electron beam). Figure 5 shows picture of the setup. All components of the prototype setup are located in light shielding box and multi-wire proportional chambers are installed both upstream and downstream of the prototype along beam line for tracking for charged particles in the beam. We take data by changing setup such as aerogel refractive index, beam incident angle, front-end electronics ASIC settings and HAPD samples. Figure 6 shows accumulative distribution of 2-dimensional hits on HAPD surface position along the beam and reconstructed Cherenkov angle of one data taking period in SPS beam test with incident angle of 0 degree. Simple estimation for separation power between $K$ and $\pi$ can be calculated by following formula:

$$
\frac{\Delta \theta_{\mathrm{C}} \sqrt{N_{\mathrm{p} . \mathrm{e}}}}{\sigma_{\mathrm{C}}}
$$

where $\Delta \theta_{\mathrm{C}}$ is the difference of Cherenkov angle between $K$ and $\pi(23 \mathrm{mrad}), N_{\text {p.e }}$ is number of detected photo electron and $\sigma_{\mathrm{C}}$ is resolution of observed Cherenkov angle. From the beam test data, we obtain $\sigma_{\mathrm{C}}=14.1 \mathrm{mrad}$ and $N_{\text {p.e }}=11.4$ then separation power is expected to be $5.5 \sigma$ for this setup. This satisfies our requirement for ARICH particle identification. For the case of non-zero incident angle, we also observe ring image and Cherenkov angle is can be reconstructed as shown in Figure 7. We take data using HAPD that has been irradiated with $\gamma$-ray and neutron. After optimizing the ASIC setting such as shaping time, offset level and so on, obtained photon yield from irradiated HAPDs bears comparison with brand-new one.

\section{Validation system for construction}

For all produced 450 HAPDs that will be used for the ARICH, we plan to check its performance before installation. Checking items so far are dark current, noise level, signal-to-noise ratio 

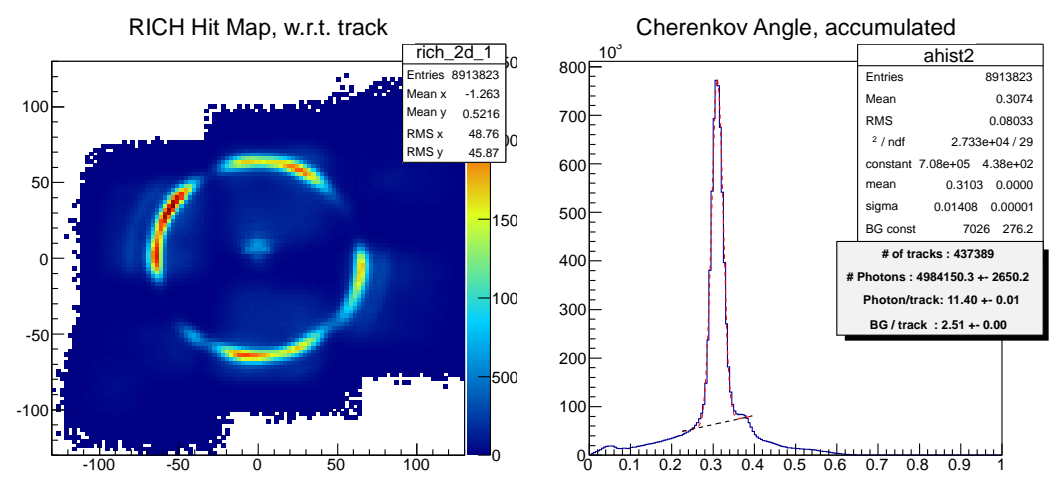

Figure 6: Accumulative distribution of 2-dimensional hits on HAPD surface position along the beam (left) and Cherenkov angle (right) in case of beam incident angle is 0 degree.
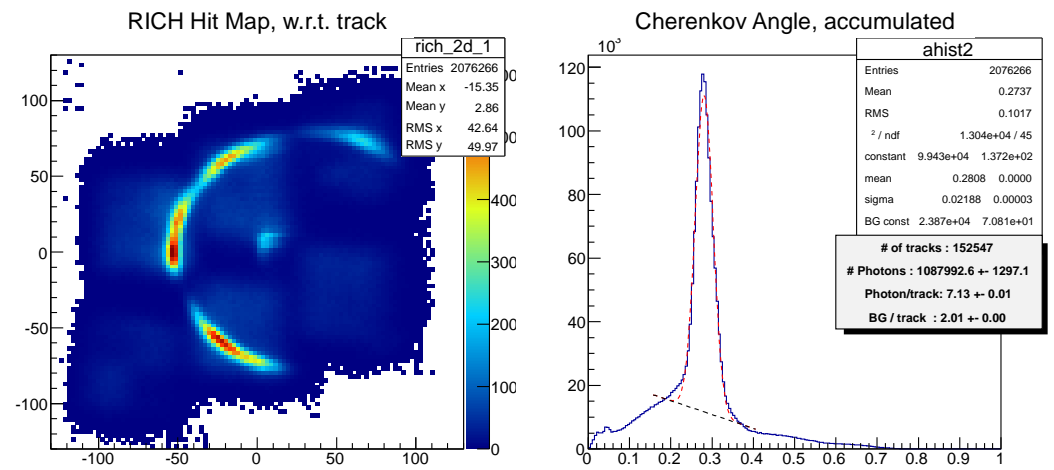

Figure 7: Accumulative distribution of 2-dimensional hits on HAPD surface position along the beam (left) and Cherenkov angle (right) in case of beam incident angle is 30 degree.

using 1 photoelectron corresponding light source, APD channel scan using laser and quantum efficiency of photocathode. The quantum efficiency is measured by comparing current with reference photodiode which quantum efficiency is known when illuminating same light to HAPD and photodiode. Validation system has been set up and start operation from September 2013 for the first lot of mass-produced HAPDs. This will be going on until all HAPD arrives in autumn 2014.

\section{Summary}

We have developed the ARICH detector as a particle identification system of Belle II experiment. To achieve the separation between $K$ and $\pi$ more than the deviation power of $4 \sigma$, we develop photon sensor HAPD which has high gain, good position resolution and radiation hardness. We set up prototype ARICH and perform test at hadron and electron beam line. Separation power for $K$ and $\pi$ is expected to be $5.5 \sigma$ from the beam test data. We start production of HAPD that will be installed in ARICH from September 2013 and the ARICH will be installed in Belle II detector in 2015.

\section{References}

[1] "Belle II Technical Design Report", T. Abe et. al., arXiv:1011.0352v1[physics.ins-det] (2010). 